\title{
Study of the peculiarities of multiparticle production via event-by-event analysis in asymmetric nucleus-nucleus interactions
}

\author{
Anastasiya Fedosimova, Adigam Gaitinov, Ekaterina Grushevskaya, and Igor Lebedev ${ }^{\mathrm{a}}$ \\ Institute of Physics and Technology, Almaty, Kazakhstan
}

\begin{abstract}
In this work the study on the peculiarities of multiparticle production in interactions of asymmetric nuclei to search for unusual features of such interactions, is performed. A research of long-range and shortrange multiparticle correlations in the pseudorapidity distribution of secondary particles on the basis of analysis of individual interactions of nuclei of ${ }^{197} \mathrm{Au}$ at energy $10.7 \mathrm{AGeV}$ with photoemulsion nuclei, is carried out. Events with long-range multiparticle correlations (LC), short-range multiparticle correlations (SC) and mixed type (MT) in pseudorapidity distribution of secondary particles, are selected by the Hurst method in accordance with Hurst curve behavior. These types have significantly different characteristics. At first, they have different fragmentation parameters. Events of LC type are processes of full destruction of the projectile nucleus, in which multicharge fragments are absent. In events of mixed type several multicharge fragments of projectile nucleus are discovered. Secondly, these two types have significantly different multiplicity distribution. The mean multiplicity of LC type events is significantly more than in mixed type events. On the basis of research of the dependence of multiplicity versus target-nuclei fragments number for events of various types it is revealed, that the most considerable multiparticle correlations are observed in interactions of the mixed type, which correspond to the central collisions of gold nuclei and nuclei of CNO-group, i.e. nuclei with strongly asymmetric volume, nuclear mass, charge, etc. Such events are characterised by full destruction of the target-nucleus and the disintegration of the projectile-nucleus on several multi-charged fragments.
\end{abstract}

\section{Introduction}

Cosmic rays, which are measured at ground stations, are a natural source of high-energy particles. The primary cosmic particle enters the Earth's atmosphere, in which numerous nuclear and electromagnetic interactions take place giving rise to a cascade of secondary particles. To determine the primary cosmic particle parameters, a complex inverse task must be solved, because a lot of interactions happen before the observation level. The most correct way for a solution of the problem is by simulating the shower. For the simulation the characteristics of the elementary act (interaction cross section, inelasticity, multiplicity of the process, etc.) from data obtained at accelerators, are used. Therefore, to understand the features of the development of cascade processes, detailed information about the fluctuations of distributions of secondary particles in different types of nucleus-nucleus interactions in the elementary act, is required [1].

Anomalous events (high multiplicity, large transverse momenta, large multiparticle correlations in pseudorapidity distributions of secondary particles, etc.) can significantly change the dynamics of the cascade process. For the research of such peculiarities in multiparticle production, different approaches and methods are used [2-6].

\footnotetext{
a e-mail: lebedev692007@yandex.ru; lebedev@sci.kz
}

A detailed event-by-event analysis, taking into account possible kinematic distinctions, can provide more information on the dynamics of the process of nucleus-nucleus interactions $[7,8]$. Analyzing in detail the characteristics of each individual event, background fluctuations can be separated from experimental peculiarities which must be taking into account to describe the development of cascade processes on the basis of interaction models.

In this work the study on peculiarities of multiparticle production in interactions of asymmetric nuclei to search for unusual features of such interactions, is performed.

In addition to the analysis of standard distributions (multiplicity, pseudorapidity, etc.), the existence of correlated groups of secondary particles and their dislocation in pseudorapidity distributions was analyzed.

For analysis of the correlated groups, Hurst's method was used $[9,10]$. To study the pseudorapidity correlations we analyzed the behaviour of the Hurst curves, which are dependent on the normalized range, $R / S$ (where $R$ and $S$ are the range and the standard deviation), versus the size of the pseudorapidity interval, $d \eta$, using the function $R / S=(a \cdot d \eta)^{\mathrm{h}}$, where $a$ is a free parameter and $h$ is the Hurst index. The analysis of the behaviour of the Hurst curve allows to estimate the "force" and "length" of multiparticle correlations in pseudorapidity distributions of secondary particles and to distinguish fluctuations of dynamically correlated distributions of secondary particles from stochastic, connected with statistical reasons [4]. 

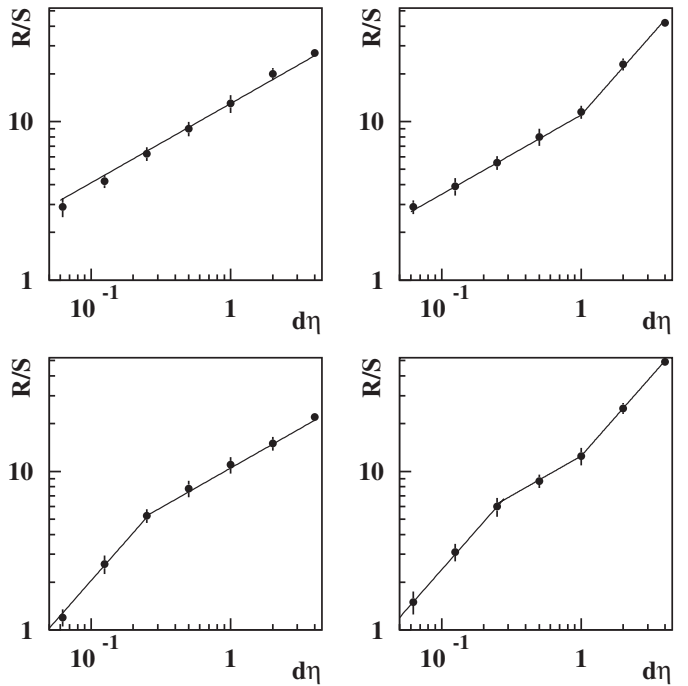

Figure 1. Behaviour of the Hurst curve for four typical events of $\mathrm{Au}+\mathrm{Em}$ 10.7 AGeV.

In the present paper we analyzed experimental pseudorapidity distributions of secondary particles obtained in 10.7 A GeV ${ }^{197} \mathrm{Au}$ interactions with emulsion nuclei [11].

Nuclear photoemulsion experiments in comparison with other approaches used for investigating nuclei interactions, is one of the most informative. At first, it has high spatial resolution. Secondly nuclear photoemulsions allow us to observe an interaction in $4 \pi$ geometry of the experiment. Most other methods have essentially dead zones in which secondary particles are not registered.

Besides, the method of nuclear emulsion allows to define the charges of fragments and provides a chance to register rather small excited nucleus-targets, and also has no energy threshold for the registration of the fragments of a nucleus-projectile.

Moreover, the emulsion technique allows us to investigate parameters of fragmentation and multiparticle processes in the same interaction.

\section{Pseudorapidity correlations}

Based on a detailed analysis of individual interactions all events are divided into four types. Characteristic events of various types are shown in Fig. 1.

The first type is characterized by the linear behavior of the Hurst curve with $h \sim 0.5$ (Fig. 1 left-upper). It corresponds to an uncorrelated pseudorapidity distribution of secondary particles. Events of such a type are well described on the basis of simulating with a stochastic pseudorapidity distribution.

Events of the second type are characterised by the Hurst index $h>0.64$ in the field of small pseudorapidity intervals and $h \sim 0.5$ at other $d \eta$ (Fig. 1 left-lower). Such behaviour of a correlation curve can be initiated by short-range multiparticle correlations. It corresponds to processes of jet type.

Events with a change of the Hurst index to $h>0.64$ in the field of large values of pseudorapidity intervals have been related to the third type (Fig. 1 right-upper). Such behaviour of the correlation curve corresponds to the essential display of long-range multiparticle correlations
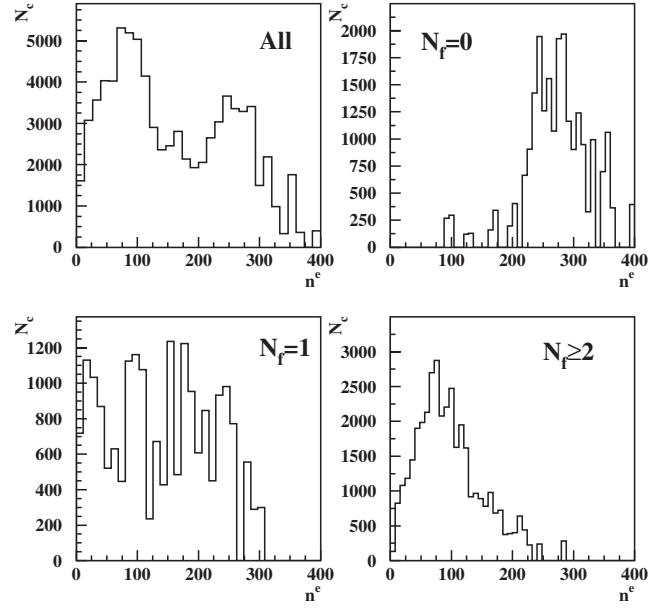

Figure 2. Multiplicative $n^{e}$-distributions for events with different numbers of multi-charge fragments in $\mathrm{Au}+\mathrm{Em} 10.7 \mathrm{AGeV}$.

and such events are referred to processes of the explosive type.

Events with $h>0.64$ at both small values and at large values of the pseudorapidity interval, but with $h \sim 0.5$ in the middle region of $d \eta$, have been referred to the fourth type (Fig. 1 right-lower). Such behaviour of the Hurst curve corresponds to events of the mixed type, including processes of the explosive and jet types.

\section{Fragmentation of the projectile nucleus}

Detailed analysis has shown that events of different types had different fragmentation parameters.

Events of the explosive type are processes of full destruction of the projectile nucleus, in which multi-charge fragments are absent. Events with several multi-charge fragments of the projectile nucleus give Hurst curves corresponding processes of the mixed type. In uncorrelated events and in processes of the jet type only one multi-charge fragment is discovered.

In addition, interesting results follow from a joint analysis of the fragmentation parameters and multiplicity distributions, which are presented in Fig. 2.

In order to reveal a deposit of low-probability (but very important) events with large multiplicity, in Fig. 2 a multiplicative distribution is presented. In this approach a weight of each event is equal to the number of particles in the event, i.e. $N_{c}=\sum n^{e}$, where $n^{e}$ is the multiplicity of an individual event.

As seen in Fig. 2, the multiplicity distribution has two clear humps (Fig. 2 left-upper).

Events of the explosive type, which are characterised by the complete absence of multi-charge fragments $N_{f}=$ 0 (Fig. 2 right-upper), have large multiplicity. They form the hump with average multiplicity $<n^{e}>\sim 272$.

Mixed type events, which have several multi-charge fragments, give their main deposit to the hump with $<n^{e}>\sim 97$ (Fig. 2 right-lower).

Events with $N_{f}=1$, which correspond to uncorrelated events and processes of the jet type, have different multiplicities in wide intervals without clearly expressed peculiarities (Fig. 2 left-lower). 


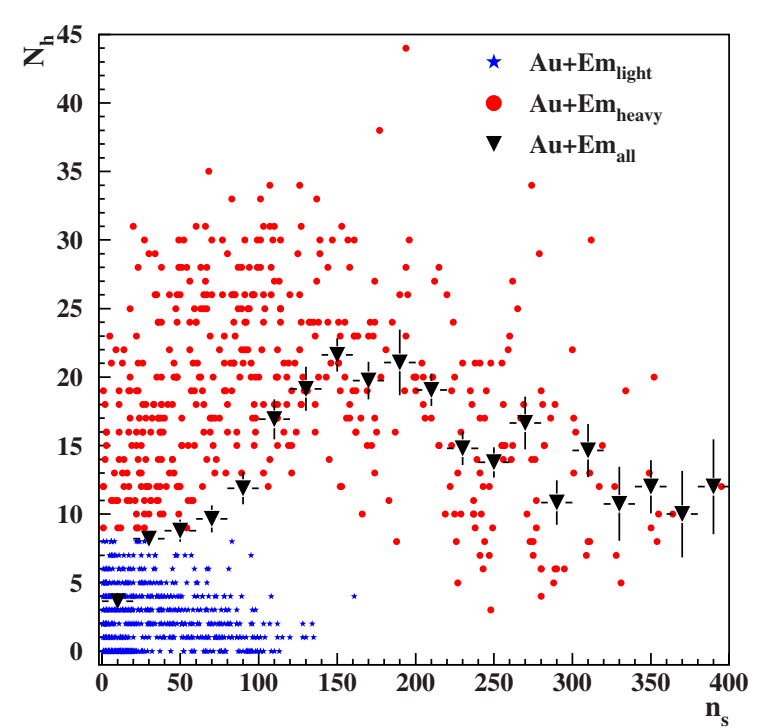

Figure 3. The distribution of events with various target-nucleus fragment number, $N_{h}$, versus multiplicity, $n_{s}$, for individual events of $A u+E m$ at $10.7 \mathrm{AGeV}$.

\section{Interactions with heavy and light nuclei}

In the considered experiments, the EMU01-collaboration $\left({ }^{197} \mathrm{Au}+\mathrm{Em} 10.7 \mathrm{AGeV}\right)$ used standard nuclear emulsions of type BR-2. It includes hydrogen (39.2\%), CNOgroup nuclei (35.3\%) and nuclei of $\mathrm{AgBr}(25.5 \%)$.

To separate events, which is connected with different asymmetries of interactions, we have analysed the dependence of the nucleus-target fragments number, $N_{h}$, versus multiplicity, $n_{s}$, for individual events of interactions of ${ }^{197} \mathrm{Au}$ with energy $10.7 \mathrm{AGeV}$ with light and heavy nuclei of photoemulsion, which are shown in Fig. 3.

As follows from Fig. 3, events of interactions with light and heavy photoemulsion nuclei are separated well enough. For interactions with light nuclei the choice is limited by two conditions.

First, the maximum number of fragments of the targetnucleus cannot exceed 8, which corresponds to the largest charge of light photoemulsion nucleus - to oxygen nucleus.

Secondly, the maximum multiplicity, $n_{s}$, in interactions with light photoemulsion nuclei is much lower in comparison with interactions with heavy photoemulsion nuclei. Use of these facts allows us to separate heavy events, which have large multiplicities and numbers of target-nucleus fragments less than 8 , from light events.

On the basis of the received separation, the dependence of the multiplicity, $n_{s}$, and the Hurst index, $h$, for individual events of interactions of ${ }^{197} \mathrm{Au} 10.7 \mathrm{AGeV}$ with heavy and light photoemulsion nuclei, is shown in Fig. 4.

The mean value of the Hurst index for interactions of gold nuclei with light photoemulsion nuclei represents a peak behaviour with a maximum in area of $n_{s}=100$, where events with the highest values of $h$ are observed.

Hence, the most considerable multiparticle pseudorapidity correlations are discovered in the central interactions of heavy nuclei of gold and $\mathrm{CNO}$-group nuclei, i.e. nuclei with strongly asymmetric volume, nuclear mass, charge, etc.

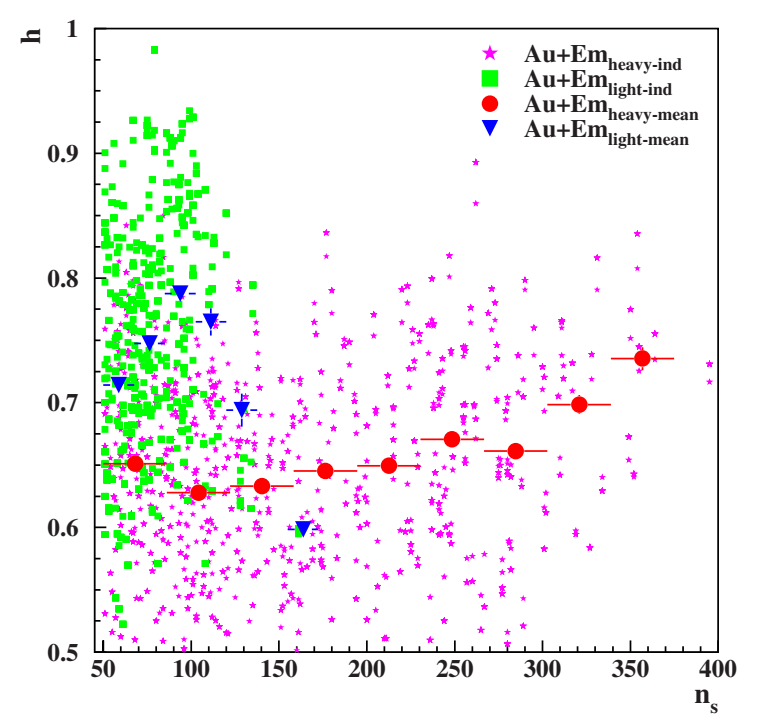

Figure 4. The distribution of events with various values of the Hurst index versus the multiplicity, $n_{s}$, for individual events of interactions of nuclei of ${ }^{197} \mathrm{Au}$ at $10.7 \mathrm{AGeV}$ with heavy and light photo-emulsion nuclei.

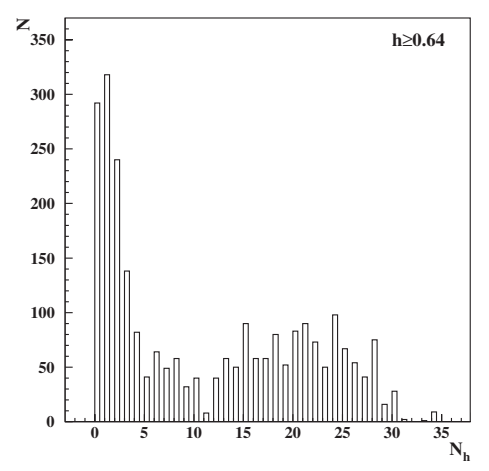

Figure 5. $N_{h}$-distribution of target-nuclei fragments for events of $\mathrm{Au}+\mathrm{Em}$ 10.7 AGeV with Hurst index $h \geq 0.64$.

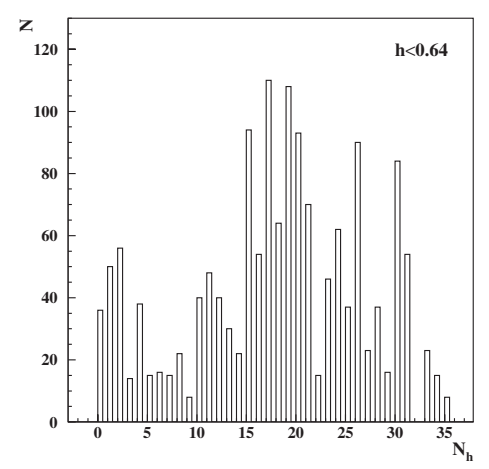

Figure 6. $N_{h}$-distribution of target-nuclei fragments for events of $\mathrm{Au}+\mathrm{Em} 10.7 \mathrm{AGeV}$ with Hurst index $h<0.64$.

\section{Fragments of the target nucleus}

Essential differences for events with $h<0.64$ and $h \geq$ 0.64 are found in distributions of target nucleus fragments. The $N_{h}$ distributions are shown in Figs. 5 and 6.

As seen in Fig. 5, the peak in the field of small values of $N_{h}$ is observed for correlation events. For events with $h<0.64$ the distribution maximum is located in average area of change of $N_{h}$ (Fig. 6). 


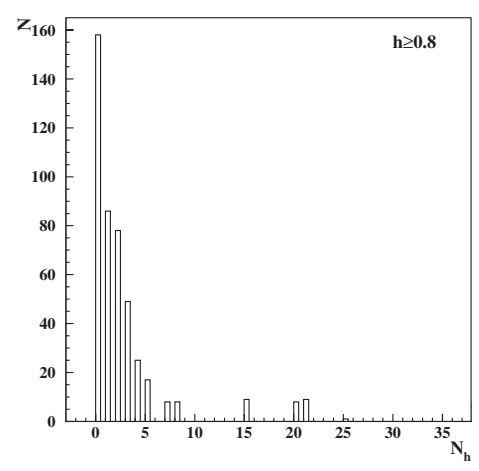

Figure 7. $N_{h}$-distribution of target-nuclei fragments for events of $\mathrm{Au}+\mathrm{Em} 10.7 \mathrm{AGeV}$ with Hurst index $h \geq 0.8$.

This effect is amplified if we consider more correlated events, i.e. by increasing the selection criterion.

The $N_{h}$-distribution for the events, selected by the criterion $h \geq 0.8$, is presented as a demonstration of the observation in Fig. 7.

As seen in Fig. 7, the majority of events with considerable multipartical correlations have in the final distribution full destruction of the target-nucleus.

\section{Conclusion}

Investigations of the density structure of pseudorapidity fluctuations in interactions of $\mathrm{Au} 10.7 \mathrm{AGeV}$ nuclei with photo-emulsion nuclei by the Hurst method, have been carried out. As a result, events with longrange multiparticle correlations, short-range multiparticle correlations and mixed type in pseudorapidity distribution of secondary particles are discovered.

Detailed analysis showed, that events of different types had different fragmentation parameters and specific multiplicity distributions.
Based on the research of the dependence of multiplicity versus target-nuclei fragment numbers for events of various types it is revealed, that the most considerable multiparticle correlations are observed in interactions of the mixed type, which correspond to central collisions of gold nuclei and nuclei of CNO-group, i.e. nuclei with strongly asymmetric volume, nuclear mass, charge, etc. Such events are characterised by the full destruction of the target-nucleus and the disintegration of the projectilenucleus into several multi-charged fragments.

This work was supported by grant N4824/GF4 of Ministry of Education and Science of Kazakhstan Republic.

\section{References}

[1] A. Haungs, Phys. Procedia 61, 425-434 (2015)

[2] M.I. Adamovich et al., Azimutal correlations of secondary particles in ${ }^{32} S$ induced interactions with $\mathrm{Ag}(\mathrm{Br})$ nuclei at $4.5 \mathrm{GeV/c/nucleon} \mathrm{Part.} \mathrm{and} \mathrm{Nucl.}$ Lett. 101, 76 (2000)

[3] M.I. Adamovich et al., Factorial Moments of ${ }^{28} \mathrm{Si}$ Induced Interactions with $\mathrm{Ag}(\mathrm{Br})$ Nuclei APH N.S. Heavy Ion Phys. 113, 213 (2001)

[4] I.A. Lebedev et al., J. Phys. G 23, 637 (1997)

[5] T.N. Kvochkina et al., J. Phys. G 26, 35 (2000)

[6] A.I. Fedosimova et al., J. Phys.: Conf. Ser. 668, 012067 (2016)

[7] S. Bhattacharya, M. Haiduc, A.T. Neagub, E. Firub, Phys. Lett. B 726, 194-205 (2013)

[8] T.K. Nayak, Int. J. Mod. Phys. E 16, 3303-3322 (2008)

[9] H.E. Hurst, R.P. Black, Y.M. Simaika, Long-Term Storage: An Experimental Study (Constable, London, 1965)

[10] J. Feder Fractals (Plenum Press, New York, 1988)

[11] M.I. Adamovich et al. Eur. Phys. J. A 5, 429-440 (1999) 